\title{
Active Power Oscillation Property Classification of Electric Power Systems Based on SVM
}

\author{
Ju Liu, ${ }^{1}$ Wei Yao, ${ }^{1}$ Jinyu Wen, ${ }^{1}$ Haibo He, ${ }^{2}$ and Xueyang Zheng ${ }^{1}$ \\ ${ }^{1}$ The State Key Laboratory of Advanced Electromagnetic Engineering and Technology, \\ Huazhong University of Science and Technology, Wuhan 430074, China \\ ${ }^{2}$ Department of Electrical, Computer, and Biomedical Engineering, University of Rhode Island, Kingston, RI 02881, USA
}

Correspondence should be addressed to Jinyu Wen; jinyu.wen@hust.edu.cn

Received 24 January 2014; Revised 21 April 2014; Accepted 23 April 2014; Published 6 May 2014

Academic Editor: Hongjie Jia

Copyright (C) $2014 \mathrm{Ju}$ Liu et al. This is an open access article distributed under the Creative Commons Attribution License, which permits unrestricted use, distribution, and reproduction in any medium, provided the original work is properly cited.

\begin{abstract}
Nowadays, low frequency oscillation has become a major problem threatening the security of large-scale interconnected power systems. According to generation mechanism, active power oscillation of electric power systems can be classified into two categories: free oscillation and forced oscillation. The former results from poor or negative damping ratio of power system and external periodic disturbance may lead to the latter. Thus control strategies to suppress the oscillations are totally different. Distinction from each other of those two different kinds of power oscillations becomes a precondition for suppressing the oscillations with proper measures. This paper proposes a practical approach for power oscillation classification by identifying real-time power oscillation curves. Hilbert transform is employed to obtain envelope curves of the power oscillation curves. Twenty sampling points of the envelope curve are selected as the feature matrices to train and test the supporting vector machine (SVM). The tests on the 16machine 68-bus benchmark power system and a real power system in China indicate that the proposed oscillation classification method is of high precision.
\end{abstract}

\section{Introduction}

Damping ratio is a key factor in electric power oscillations. With the interconnection of large-scale power grids via high voltage long distance transmission lines, coupling between synchronous generators becomes weaker which leads to poor or negative damping ratio of power systems $[1,2]$. Power oscillation has become a major problem threatening the security of large-scale interconnected power systems $[3,4]$. In order to safely operate the power system, monitoring classification and suppression of the electric power oscillations in power grids has captured exponentially increasing attention in power engineering community in the past decade.

This paper focuses on the active power oscillation property classification problem. According to generation mechanism, active power oscillations of electric systems can be classified into two categories [2]. One is free oscillation resulted by negative damping ratio of power systems. It could also be called negative damping oscillation. Worldwide accepted explanation mechanism of this kind of power oscillation is based on the complex torque analysis method proposed by DeMello and Concordia in 1969 [5]. When the reactance of transmission system is large or power output of generators is high, the negative damping torque produced by lagging phase of quick excitation circuit counteracts the original positive damping of generators' damping windings. This will lead to negative damping ratio of power grids and cause a power oscillation with increasing amplitude [6,7]. Reducing transmission power of tie lines or installing PSS equipment which enhances damping torque through phase compensation has been proved to be important measure for suppressing negative damping power oscillation $[8,9]$.

The other kind of active power oscillation is forced power oscillation, which is explained by the resonance mechanism $[10,11]$. Power resonance of generators will be provoked when the frequency of a small periodic disturbance occurring at the power system is equal or close to the system's natural frequency. This kind of power oscillation is characterized by fast oscillation start and rapid decay after losing oscillation source. Some researchers have demonstrated that periodic 
disturbances of excitation circuit, turbine speed governor system, and active power load could stimulate forced power oscillation [12-14]. Separating the periodic disturbance sources from the system is the most effective countermeasure to eliminate its influence quickly [15].

Thus negative damping oscillation and forced oscillation have different generation mechanisms with different coping measures. It is extremely important and necessary to distinguish them from each other. However, both of them have similar oscillation forms with increasing amplitudes at initial stage and probably develop into a constant amplitude oscillation in the end. Correct and rapid identification of the oscillation property becomes a difficult problem to be solved. Nowadays, researches on power oscillation classification mostly concentrate on simulation after oscillation accidents. System response curves of simulation under negative damping oscillation condition and forced oscillation condition are compared with the actual oscilloscope records of power systems to judge the oscillation property of power oscillation accidents $[16,17]$. An online oscillation property classification method based on difference analysis of the oscillation curves is proposed in [18], but there is a certain error of the differential calculation. A fundamental theory of forced power oscillation in a power system has been recommended in [19], which has proved that the forced power oscillation has different envelop curve from the negative damping oscillation. Thus the active power oscillation property could be identified online by distinguishing their envelop curves.

Statistical learning theory and support vector machine (SVM) have given a systemic theoretical explanation about pattern recognition under circumstances of finite samples. Many problems, like model-choosing, overfitting, nonlinear, disaster of dimensionality, and local minimum, which have long hindered the development of machine learning are now solved to a great extent $[20,21]$. So far, SVM has successfully been applied to many fields like fault diagnosis, speech recognition, image recognition and text classification [22-24]. In [25], two types of SVM were implemented to effectively classify different kinds of power quality disturbances.

This paper proposes a practical approach for power oscillation classification by recognition of real-time power oscillation curves utilizing SVM. Hilbert transform is employed to obtain envelope curves of the power oscillation curves. Twenty sampling points on the envelope curve of power oscillation are selected for feature extraction. Then, forty power oscillation curves are employed as samples to train the supporting vector machine. At last, three tests on the 16-machine 68-bus benchmark system and a real power system in China indicate that the proposed oscillation classification method possesses good precision.

The rest of this paper is organized as follows. In Section 2, a Hilbert transform based oscillation feature extraction scheme is proposed. Section 3 introduces a power oscillation property classification method utilizing SVM. Case study is undertaken on the 16-machine 68 -bus benchmark system and a practical simplified power system of China to verify the effectiveness of the proposed oscillation property classification method in Section 4. Conclusions are given in Section 5 .

\section{Feature Extraction of Power Oscillations}

2.1. Introduction of Power Oscillations. When a free oscillation happens in a power system, the rotor angle, rotation speed of synchronous generators, and relevant electric variables (such as the power flow of transmission lines and bus voltages) will oscillate accordingly, among which the power flow of transmission line $P_{i j}$ can be expressed as follows:

$$
P_{i j}=\sum_{i=1}^{n} k_{i} x_{i 0} e^{\lambda_{i} t},
$$

where $k_{i}$ denotes the participation factor of oscillation pattern $i ; x_{i 0}$ is the initial value of state variable according to oscillation pattern $i ; \lambda_{i}$ denotes the eigenvalue of the oscillation pattern $i$. Generally $\lambda_{i}$ can be expressed as

$$
\lambda_{1,2}=\alpha \pm j \omega .
$$

In (2), $\alpha$ indicates the damping performance of the oscillation; $\omega$ denotes the frequency characteristic of the oscillation; the damping ratio of the oscillation is defined as

$$
\xi=\frac{-\alpha}{\sqrt{\alpha^{2}+\omega^{2}}} .
$$

The oscillation curve of any free oscillation mode can be expressed as

$$
P_{i j}=B_{1} e^{\alpha t} \sin \left(\omega t+\phi_{1}\right) .
$$

Therefore, when any oscillation mode with $\xi<0$ (i.e., $\alpha>0$ ) exists in the power system, any small disturbance would stimulate this mode and result in a power oscillation of the tie line with increasing amplitude as shown in Figure 1(a). That is the so-called negative damping oscillation. If all the damping ratios of oscillation patterns are larger than zero, the small disturbance occurring at the power system would be restrained by the positive damping.

For any forced oscillation in the power system with an extreme disturbance like $h \sin \left(\omega_{d} t\right)$, it can be expressed as a second-order system:

$$
\ddot{x}+2 \xi \omega \dot{x}+\omega^{2} x=h \sin \left(\omega_{d} t\right) .
$$

Solving (5), the oscillation curve of forced oscillation is

$$
P_{i j}=B_{1} e^{\alpha t} \sin \left(\omega t+\phi_{1}\right)+B_{2} \sin \left(\omega_{d} t+\phi_{2}\right) .
$$

Under the circumstance of $\xi>0$ (i.e., $\alpha<0$ ), the free oscillation part $B_{1} e^{\alpha t} \sin \left(\omega t+\phi_{1}\right)$ in (6) will decay. But power resonance would be motivated and large oscillation would follow as shown in Figure 1(b) if the oscillation frequency $\omega_{d}$ of the extreme disturbance is equal or close to the system's natural frequency $\omega$.

From the analysis above, it can be concluded that the envelope curve of negative damping oscillation increases as $B_{1} e^{\alpha t}$. The envelope curve of forced oscillation will increase at the initial stage. Then it transits to an oscillation as $(h / 2(\omega-$ $\left.\left.\omega_{d}\right) \omega\right) e^{\alpha t} \sin \left(\left(\omega-\omega_{d}\right) t+\phi_{2}\right)+B_{2}$ at the transitory stage. At last it will die down to a constant as $B_{2}$ at stable stage [19]. Thus there is obvious distinction between the envelope curves of the two kinds of power oscillations. 


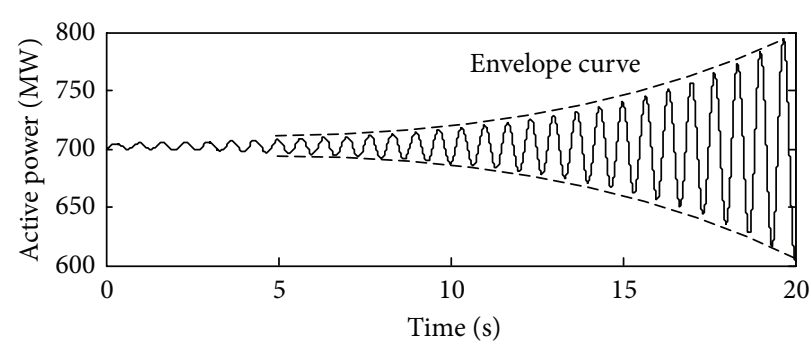

(a) Negative damping oscillation

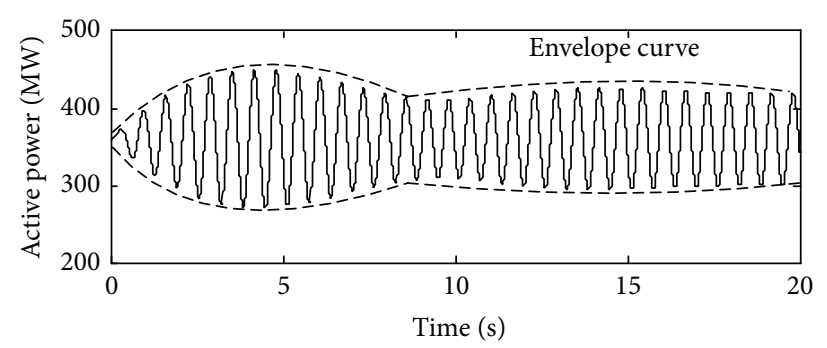

(b) Forced oscillation

Figure 1: Power oscillation curve of tie line.

2.2. Hilbert Transform. Hilbert transform (HT) [26] offers an effective approach to extract envelope curves as oscillation features for different kinds of power oscillations. For the following power oscillation signal in a power grid:

$$
u(t)=A(t) \cos \varphi(t) .
$$

HT could be applied to acquire its complex conjugate signal

$$
v(t)=H[u(t)]=\frac{1}{\pi} \int_{-\infty}^{+\infty} \frac{u(\tau)}{t-\tau} d \tau .
$$

$v(t)$ is a sinusoidal signal similar to $u(t)$. It could be expressed as $v(t)=A(t) \sin \varphi(t) \cdot u(t)$ and $v(t)$ constitute a HT pair which makes up the following HT analytic signal:

$$
w(t)=A(t) \cos \varphi(t)+j A(t) \sin \varphi(t) .
$$

Namely,

$$
\begin{aligned}
& w(t)=A(t) e^{j \varphi(t)}, \\
& A(t)=\sqrt{u^{2}(t)+v^{2}(t) .}
\end{aligned}
$$

Therefore, the amplitude $A(t)$ of HT analytic signal reflects global change trend of the signal. $A(t)$ represents the envelope of the oscillation signal and could be calculated by using (8) when the analytic signal is a signal with intrinsic oscillation mode.

2.3. Feature Extraction. The envelope curve of power oscillation will be obtained by HT after the power oscillation in power grid is detected by the wide area measurement system (WAMS). Then the envelope curve is normalized according to the steady value before oscillation happens. Finally, twenty evenly spaced points on the envelope curve are selected in every $2 T$ interval ( $T$ is oscillation period of the power oscillation) to constitute a group of feature matrices as shown in Figure 2.

It is well known that the power oscillation in a power system generally combines various swing modes. If there is only one dominant oscillation mode, the oscillation amplitude of other oscillation modes is small compared with the dominant oscillation mode. The influence of other oscillation modes on dominant oscillation mode can be neglected. If there are two or more than two dominant oscillation modes, the dominant

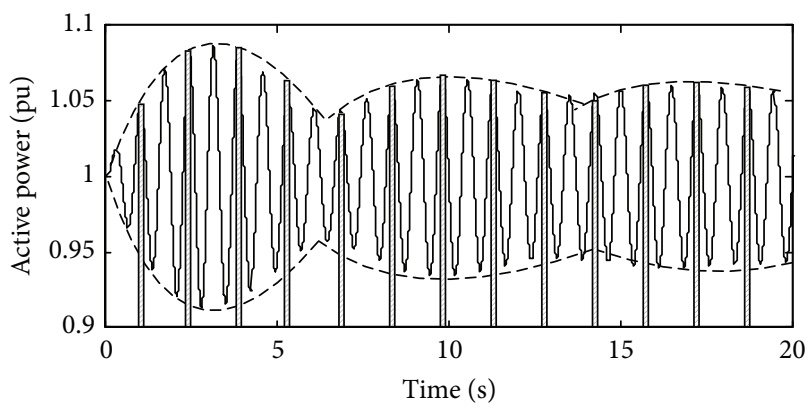

FIGURE 2: Extraction of the elements in the feature matrices.

oscillation mode with positive damping ratio will decay after seconds or minutes. Then only the oscillation mode with negative damping ratio retains. Meanwhile, the data used for power oscillation feature extraction contains 40 cycles of the oscillation curve. After several cycles, only the oscillation mode with negative damping ratio will retain and be used for the oscillation property classification.

However, the power oscillation with two negative damping oscillation modes has similar expression with forced power oscillation. The beat-frequency oscillation will also be perceived in the power oscillation with two negative damping oscillation modes. It is hard to distinguish the forced power oscillation and the power oscillation with two negative damping oscillation modes. However, the power oscillation with two negative damping oscillation modes is very unusual in power system; thus the negative damping power oscillation in this paper is the power oscillation with only one negative damping oscillation mode. Under this premise, the feature matrices can represent the power oscillation property of the system.

Difference of adjacent points of the feature matrices denotes the change direction of the envelope curve and the second-order difference denotes its change tendency. As for negative damping oscillation, the variation rate of the envelope curves is generally positive with oscillation amplitude growing more and more rapidly. This means that the firstorder and second-order difference of adjacent points from the feature matrices should also be positive. However, for forced oscillations, the envelope curve has minimum and maximum points. This indicates that the first-order or second-order difference of adjacent points could be zero or negative. 


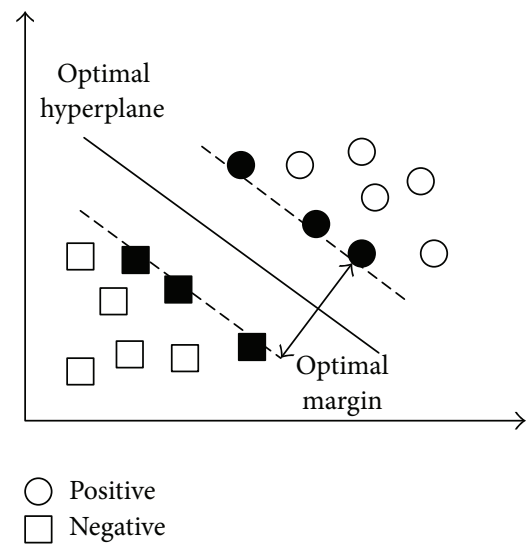

FIgURE 3: A classification example in a two-dimensional space based on SVM $[27,28]$.

Therefore, the feature matrices obtained in this paper at least contain the oscillation characteristic of the power oscillation which could be utilized for power oscillation property classification. Besides, the feature matrices extraction of power oscillation curves will be the foundation of the following work.

\section{Oscillation Property Classification Based on SVM}

3.1. Basic Theory of SVM. SVM-based classification method is established on the basis of structural risk minimization principle and VC theory (Vapnik and Chervonenkis theory) [27]. In order to obtain excellent capability of generalization according to a certain amount of samples, the SVM-based classification method seeks the best compromise between the complication of the model and its learning capacity. The principle idea of SVM is as follows: it firstly maps the input vectors in sample space into some high or even infinite dimensional feature space through some nonlinear mapping function $\phi(x)$. And then the nonlinear and nonclassifiable problem in the original sample space is transformed into a linear and classifiable problem in the high dimensional feature space. At last, in this feature space, a linear decision surface is constructed with special properties that possess the largest separation margin between the two classes just as shown in Figure 3.

For the given linear and separable training patterns $\left\{\left(x_{i}, d_{i}\right)\right\}_{i=1}^{l}, x_{i} \in R^{n}, d_{i} \in\{1,-1\}, x_{i}$ denotes the $n$ dimensional input vector and $d_{i}$ denotes the class of the sample. For further explanation, 1 denotes the positive class and -1 denotes the negative class. These two sets are linearly separable on the condition that there are a vector $\omega$ and scalar $b$ which satisfy

$$
\omega \cdot x_{i}+b \geq 1,
$$

if $x_{i} \in$ positive sample set, namely $y_{i}=1$,

$$
\omega \cdot x_{i}+b \leq-1,
$$

if $x_{i} \in$ negative sample set, namely $y_{i}=-1$.
If the vector $\omega$ has the minimum norm, then the hyperplane $y=\omega \cdot x+b$ separates the training data with a maximal margin. It is the unique and optimal hyper plane for classification of the train data.

If the training data in the input space is nonlinear, in order to construct a hyper plane to classify the nonlinear samples, one first has to map the input vector $x$ into a higher dimension feature space by function $\phi(x)$ and then takes the sign of the function

$$
y=\omega \cdot \phi(x)+b .
$$

Consider the case when the training data cannot be classified without error. Penalty constant $C$ and some nonnegative variables $\xi_{i}$ are introduced to punish the incorrect classification. Then this idea can be expressed formally as an optimization problem as follows:

$$
\begin{array}{ll}
\min & \frac{1}{2}\|\omega\|^{2}+C \sum_{i=1}^{l} \xi_{i} \\
\text { s.t. } & d_{i}\left[\omega \cdot \phi\left(x_{i}\right)+b\right]+\xi_{i} \geq 1 \\
& \xi_{i} \geq 0 .
\end{array}
$$

The Lagrange function for this problem is

$$
\begin{aligned}
& L(\omega, b, \xi, \Lambda, \mathrm{R}) \\
& =\frac{1}{2}\|\omega\|^{2} \\
& \quad+C \sum_{i=1}^{l} \xi_{i}-\sum_{i=1}^{l} \alpha_{i}\left(d_{i}\left[\omega \cdot \phi\left(x_{i}\right)+b\right]+\xi_{i}-1\right) \\
& \quad-\sum_{i=1}^{l} r_{i} \xi_{i},
\end{aligned}
$$

where the nonnegative multipliers $\Lambda^{T}=\left(\alpha_{1}, \alpha_{2}, \ldots, \alpha_{l}\right)$ and $R^{T}=\left(r_{1}, r_{2}, \ldots, r_{l}\right)$ arise from the constraint in (13).

Using the conditions for the minimum of this function at the extreme point

$$
\begin{gathered}
\left.\frac{\partial L}{\partial \omega}\right|_{\omega=\omega_{0}}=\omega_{0}-\sum_{i=1}^{l} \alpha_{i} d_{i} \phi\left(x_{i}\right)=0, \\
\left.\frac{\partial L}{\partial b}\right|_{b=b_{0}}=\sum_{i=1}^{l} \alpha_{i} d_{i}=0, \\
\left.\frac{\partial L}{\partial \xi_{i}}\right|_{\xi_{i}=\xi_{i 0}}=C-\alpha_{i}-r_{i}=0 .
\end{gathered}
$$

From (15), $\omega_{0}$ can be obtained as

$$
\omega_{0}=\sum_{i=1}^{l} \alpha_{i} d_{i} \phi\left(x_{i}\right) .
$$

Thus the decision function in (12) is transformed into the following form:

$$
y=\sum_{i=1}^{l} d_{i} \alpha_{i} K\left(x, x_{i}\right)+b .
$$


Substituting the expressions for $\omega_{0}, b_{0}$, and $\xi_{i 0}$ to the Lagrange function (13),

$$
F(\Lambda)=\sum_{i=1}^{l} \alpha_{i}-\frac{1}{2} \sum_{i=1}^{l} \sum_{j=1}^{l} \alpha_{i} \alpha_{j} d_{i} d_{j} K\left(x_{i}, x_{j}\right) .
$$

The original convex optimization problem is also transformed into a quadratic optimization problem:

$$
\begin{aligned}
\min _{\Lambda} & F(\Lambda)=\sum_{i=1}^{l} \alpha_{i}-\frac{1}{2} \sum_{i=1}^{l} \sum_{j=1}^{l} \alpha_{i} \alpha_{j} d_{i} d_{j} K\left(x_{i}, x_{j}\right) \\
\text { s.t. } & \sum_{i=1}^{l} \alpha_{i} d_{i}=0 \\
& 0 \leq \alpha_{i} \leq C .
\end{aligned}
$$

Under this circumstance, the optimal Lagrange's multiplicator $\alpha_{i}^{0}$ can be obtained by using the Kuhn-Tucker condition in quadratic programming problem. As for the optimal hyperplane algorithm, the vector $\omega$ can be written as a combination of the training data

$$
\omega_{0}=\sum_{i=1}^{l} d_{i} \alpha_{i}^{0} \phi^{T}\left(x_{i}\right)
$$

According to (21) and (18), the classification function of the training data can be obtained as

$$
y=\operatorname{sgn}\left[\sum_{i=1}^{l} d_{i} \alpha_{i}^{0} \phi^{T}\left(x_{i}\right) \phi(x)+b\right] .
$$

From (12)-(22), $\xi_{i}$ denotes the slack variable and $K\left(x_{i}, x_{j}\right)$ is the kernel function. $K\left(x_{i}, x_{j}\right)$ is the convolution of dot product by $\phi\left(x_{i}\right)$ and $\phi\left(x_{j}\right)$ in the feature space:

$$
K\left(x_{i}, x_{j}\right)=\phi^{T}\left(x_{i}\right) \phi\left(x_{j}\right) .
$$

The input parameters of the kernel function are the training data $x_{i}$ of power system, and the kernel function for the SVM is independent of the power system. Any function that satisfies Mercer's condition can be used as kernel function [27]. By employing different kinds of kernel functions, different learning machines with arbitrary types of decision surface can be constructed. The most commonly used three kernel function will be employed in this paper [28]:

linear function:

$$
K\left(x_{i}, x_{j}\right)=\left(x_{i} \cdot x_{j}+1\right)^{d},
$$

sigmoid function:

$$
K\left(x_{i}, x_{j}\right)=\tanh \left(x_{i} \cdot x_{j}-\theta\right),
$$

radial basis function:

$$
K\left(x_{i}, x_{j}\right)=\exp \left(-\frac{\left|x_{i}-x_{j}\right|^{2}}{2 \sigma^{2}}\right) .
$$

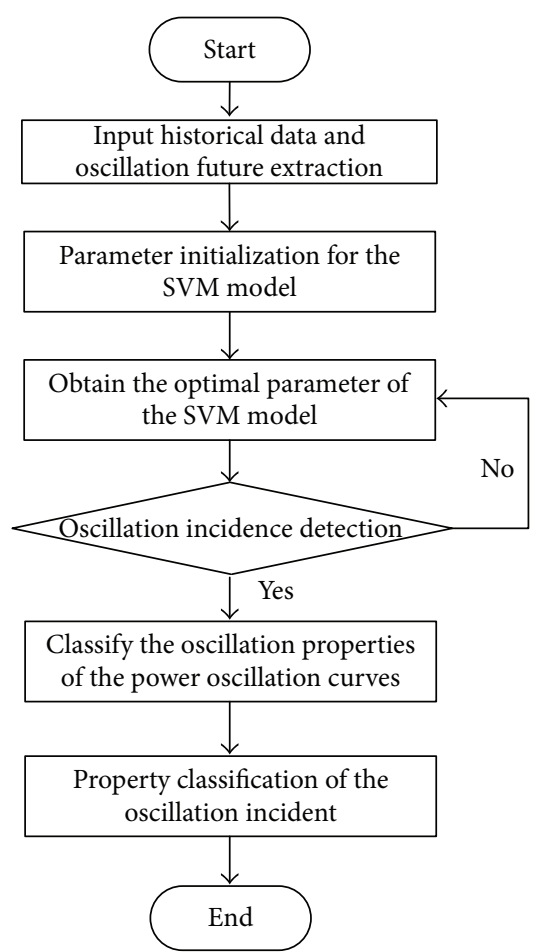

Figure 4: Flow chart of the oscillation property classification.

3.2. Procedures of the Oscillation Property Classification. When power oscillation happens, active power oscillations at substation, tie line, and generator terminal will be detected by WAMS. The oscillation property can be well classified by training historical data with SVM algorithm. Figure 4 gives the flow chart of the oscillation property classification. The following procedures will be employed.

(1) Input historical data and oscillation future extraction. Collect the power oscillation data of the power oscillation incidence that has happened. And then extract the oscillation feature of the power oscillation data by the proposed method in Section 2 as the training data for the SVM.

(2) Parameter initialization for the SVM model: Lagrange multiplier $\alpha$ and threshold $b$ are assigned with random number.

(3) Obtain the optimal parameter of the SVM model. Based on training samples, the objective function of (20) is established and the optimal problem is solved using Kuhn-Tucker condition to obtain the optimal $\alpha$ and $b$.

(4) Power oscillation incidence detection: judge whether the power oscillation amplitude of the fifth cycle is larger than $50 \mathrm{MW}$ or not for any $500 \mathrm{kV}$ tie line and record the oscillation curve for the oscillation property classification.

(5) Classify the oscillation properties of the power oscillation curves. Classify the property of the power oscillation curves recorded by the WAMS from different 


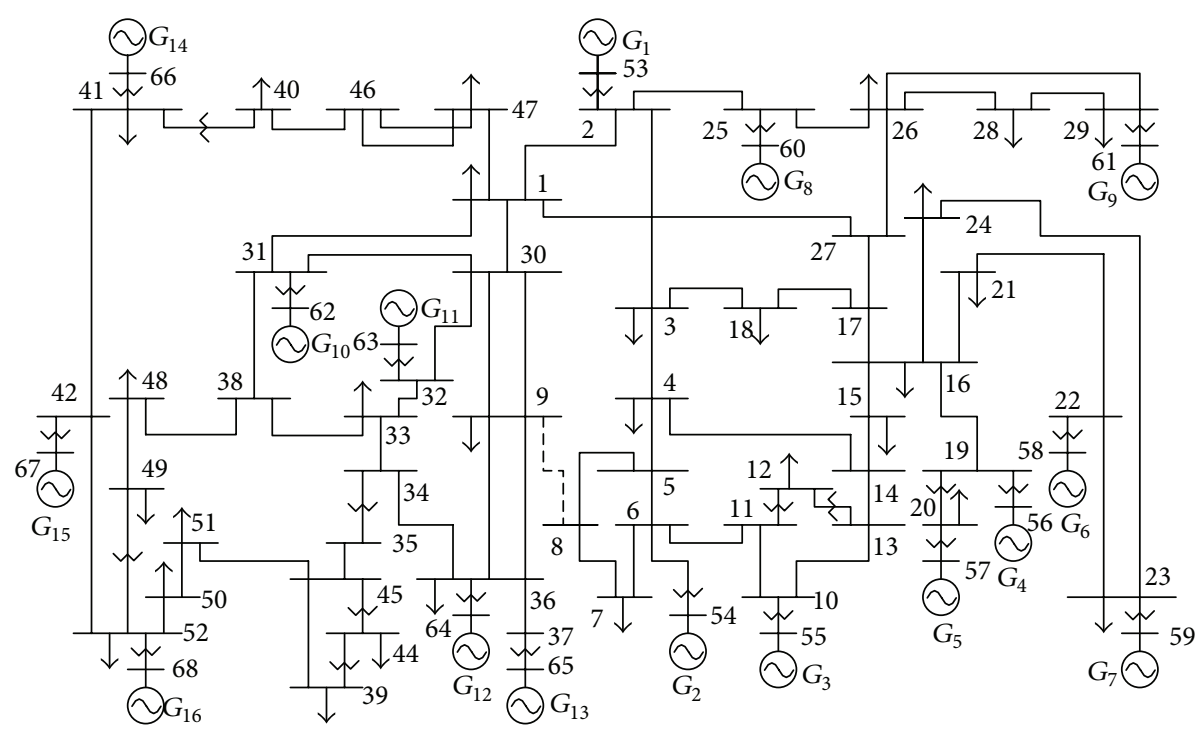

FIGURE 5: Simulation example of IEEE16-machine 68-bus benchmark system.

buses at the power grid using the trained SVM model in procedure (3). As for the recognized result of the oscillation curves, 1 represents the negative damping oscillation and -1 represents the forced oscillation.

(6) Property classification of the oscillation incident: if the quantity of 1 is much more than -1 , the oscillation incident is negative damping oscillation. Else if the quantity of -1 is much more than 1 , the oscillation incident is forced oscillation.

\section{Simulation Verification}

4.1. Tests on 16-Machine 68-Bus System. The circuit diagram of IEEE16-machine 68-bus benchmark system is shown in Figure 5. Some forced oscillating sources with oscillation frequency close to the natural oscillation frequency of the generators are applied at different nodes in the power grid and ninety-six samples of power oscillation curves are obtained. Then sixty-four power oscillation curves under the negative damping condition are obtained by lowering the gain of power system stabilizer (PSS). Twenty forced power oscillation curves (represented by $\mathrm{C} 1$ ) and negative damping power oscillation curves (represented by C2), respectively, are selected for training and the remaining 120 oscillation curves are used for testing. Table 1 gives the simulation result for the oscillation property classification based on the proposed method. A $2 \times 2$ confusion matrix is constructed to show the classification performance for each case. The diagonal elements represent the correctly classified power oscillation types. The off-diagonal elements represent the misclassifications. As we can see from Table 1, different kernel function for SVM model will bring different classification precision for power oscillation curves. When sigmoid function is selected as kernel function, the model has highest accuracy. Therefore, this paper adopts this function as kernel function for SVM model.
Since noise is omnipresent in electrical power system, Gaussian white noise is considered in the classification of power oscillations. Different levels of noises with the noise to signal ratio values ranging from $5 \%$ to $10 \%$ were considered. As the noise to signal ratio increases, the adaptability of SVM model decreases and the precision of the power oscillation property classification degrades. Table 1 shows that the precision of the power oscillation property classifycation is still above $88 \%$ even with the noise to signal ratio rising up to $10 \%$. Thus the SVM-based proposed method can effectively classify different kinds of the power oscillation.

4.2. Analysis of Forced Power Oscillation in a Real Incident. In 2008, a thermal power plant was asynchronously connected to the Central China Power Grid through $110 \mathrm{kV}$ transmission lines. Power oscillations were observed in Henan, Hunan, and Jiangxi power grids. The whole oscillation duration was about two minutes with $0.7 \mathrm{~Hz}$ oscillation frequency at initial stage and then the oscillatory power of the tie line increased gradually. When the oscillation stabilized, the oscillatory frequency of the power grid was $0.62 \mathrm{~Hz}$ and the oscillatory amplitude of the active power of tie line between Henan and Hubei was about $250 \mathrm{MW}$. Subsequently, the $110 \mathrm{kV}$ transmission line in the fault region was cut off and the oscillation died down quickly. This power oscillation incident was classified as a typical forced power oscillation incident and the thermal power plant was the oscillation source [29]. The active power oscilloscope record of Yao-Shao tie line is given in Figure 6. Eleven additional active power oscilloscope records of different tie lines are selected for the oscillation property classification. The support vector machine trained at Section 4.1 is employed to identify the oscillation property of the eleven active power oscilloscope records and the result is shown in Table 2 . It can be seen that only one power oscillation curve is misidentified as negative damping oscillation and the other ten oscillation curves are all identified as forced oscillation. 
TABLE 1: Testing result of 16-machine 68-bus benchmark system.

\begin{tabular}{|c|c|c|c|c|c|c|c|c|c|}
\hline \multirow{2}{*}{ Kernel function } & \multicolumn{9}{|c|}{ Signal type } \\
\hline & \multicolumn{3}{|c|}{ No noise } & \multicolumn{3}{|c|}{$5 \%$ noise } & \multicolumn{3}{|c|}{$10 \%$ noise } \\
\hline \multirow{4}{*}{ Polynomial kernel function } & & $\mathrm{Cl}$ & $\mathrm{C} 2$ & & $\mathrm{C} 1$ & $\mathrm{C} 2$ & & $\mathrm{C} 1$ & $\mathrm{C} 2$ \\
\hline & $\mathrm{C} 1$ & 72 & 1 & $\mathrm{C} 1$ & 70 & 3 & $\mathrm{Cl}$ & 66 & 4 \\
\hline & $\mathrm{C} 2$ & 4 & 43 & $\mathrm{C} 2$ & 6 & 41 & $\mathrm{C} 2$ & 10 & 40 \\
\hline & Precision & \multicolumn{2}{|c|}{$95.80 \%$} & Precision & \multicolumn{2}{|c|}{$92.50 \%$} & Precision & \multicolumn{2}{|c|}{$88.30 \%$} \\
\hline \multirow{4}{*}{ RBF kernel function } & & $\mathrm{C} 1$ & $\mathrm{C} 2$ & & $\mathrm{C} 1$ & $\mathrm{C} 2$ & & $\mathrm{C} 1$ & $\mathrm{C} 2$ \\
\hline & $\mathrm{C} 1$ & 74 & 2 & $\mathrm{C} 1$ & 71 & 2 & $\mathrm{Cl}$ & 66 & 4 \\
\hline & C2 & 2 & 42 & $\mathrm{C} 2$ & 5 & 42 & $\mathrm{C} 2$ & 10 & 40 \\
\hline & Precision & \multicolumn{2}{|c|}{$96.70 \%$} & Precision & \multicolumn{2}{|c|}{$94.20 \%$} & Precision & \multicolumn{2}{|c|}{$88.30 \%$} \\
\hline \multirow{4}{*}{ Sigmoid kernel function } & & $\mathrm{C} 1$ & $\mathrm{C} 2$ & & $\mathrm{Cl}$ & $\mathrm{C} 2$ & & $\mathrm{C} 1$ & $\mathrm{C} 2$ \\
\hline & $\mathrm{Cl}$ & 75 & 1 & $\mathrm{C} 1$ & 72 & 1 & $\mathrm{Cl}$ & 68 & 3 \\
\hline & $\mathrm{C} 2$ & 1 & 43 & $\mathrm{C} 2$ & 4 & 43 & $\mathrm{C} 2$ & 8 & 41 \\
\hline & Precision & \multicolumn{2}{|c|}{$98.30 \%$} & Precision & \multicolumn{2}{|c|}{$95.80 \%$} & Precision & \multicolumn{2}{|c|}{$90.80 \%$} \\
\hline
\end{tabular}

TABLE 2: Identification result of oscilloscope records of forced oscillation incidence.

\begin{tabular}{lccc}
\hline Oscillation type & $\begin{array}{c}\mathrm{C} 1 \\
\text { (forced oscillation) }\end{array}$ & $\begin{array}{c}\mathrm{C} 2 \\
\text { (negative damping oscillation) }\end{array}$ & Identification result \\
\hline Number & 10 & 1 & $\mathrm{C} 1$ \\
\hline
\end{tabular}

Considering identification error, forced oscillation is chosen as the final identification result, which is consistent with the analytical result of the incident [29].

4.3. Analysis of Negative Damping Oscillation in a Real Incident. In 2005, a wide area power oscillation took place in Central China Power Grid and the whole oscillation lasted for about five minutes. The power oscillation frequency was about $0.77 \mathrm{~Hz}$. Large power fluctuations were detected at the Three Gorges Hydroelectric Power Plant and its nearby power plants in Douli, Jianglin, Longquan, and Wanxian. Then the operator of the power grid increased the output reactive power of the Three Gorges Hydroelectric Power Plant and decreased the active power output of Huanglongtan Power Plant in northwest Hubei. Subsequently, the oscillation decayed gradually until it died out. The incident was a typical negative damping power oscillation [18]. Figure 7 shows the active power oscilloscope record of the second Long-Dou tie line during the power oscillation. Twenty active power oscilloscope records of different tie lines at the power grid are selected to identify the oscillation property by the SVM trained in Section 4.1. The identification result is shown in Table 3. Taking identification error into consideration, the result in Table 3 indicates that the oscillation incident belongs to negative damping oscillation, which is also consistent with the analysis result [18].

\section{Conclusions}

Active power oscillation in power systems can be classified into two types: negative damping oscillation and forced oscillation. Although their oscillation curves are similar, the adopted suppressing control strategies are totally different.

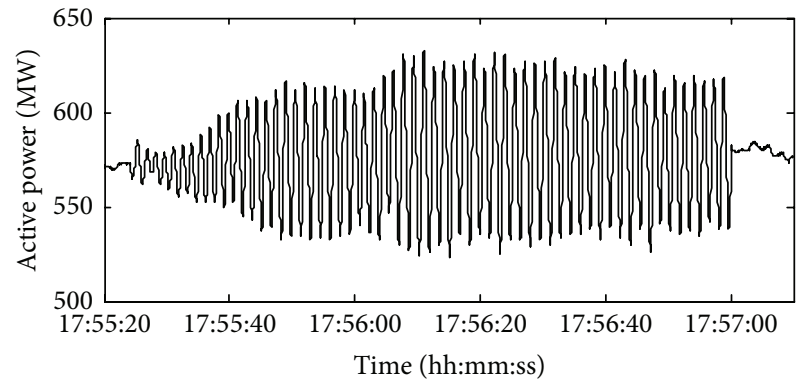

FIGURE 6: The active power oscilloscope record of Yao-Shao tie line.

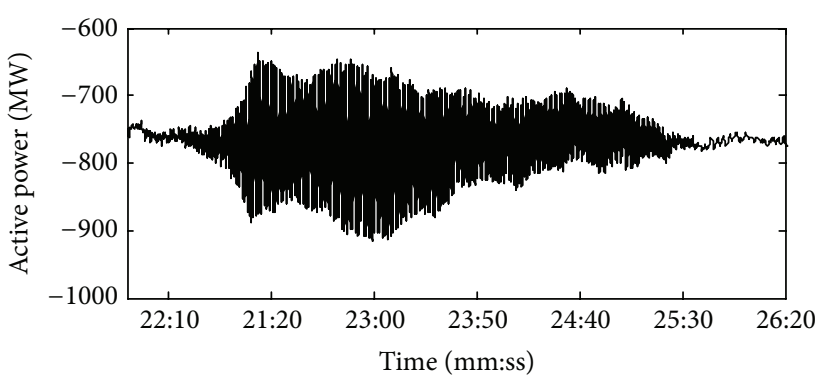

FIGURE 7: The active power oscilloscope record of the second LongDou tie line.

Thus the oscillation property classification is the premise for suppressing the power oscillation. To properly identify the oscillation property, the paper proposed an oscillation property classification method utilizing the SVM. Through the simulation analysis, it can be concluded that the envelope curves of the negative damping oscillation and forced oscillation are different and can be used as the feature for classification. As noise is omnipresent in electrical power system, 
TABLE 3: Identification result of oscilloscope records of negative damping oscillation incidence.

\begin{tabular}{lccc}
\hline Oscillation type & $\begin{array}{c}\mathrm{C} 1 \\
\text { (forced oscillation) }\end{array}$ & $\begin{array}{c}\mathrm{C} 2 \\
\text { (negative damping oscillation) }\end{array}$ & Identification result \\
\hline Number & 2 & 18 & C2 \\
\hline
\end{tabular}

Gaussian white noise is considered in the research of power oscillation classification. With the increase of the noise to signal ratio, the adaptability of SVM model decreases and the precision of the power oscillation property classification degrades. However, even with the noise to signal ratio reaching up $10 \%$, the precision of the power oscillation property classification is still above $88 \%$. Moreover, two real incidents in the real power systems are used to verify the proposed classification method. Results show that the identified results from the proposed method is the same with the postincident analytical identification result, while the postanalytical identification is an offline time consuming method only applied after the oscillation happens. The proposed method can be applied online and provides guidance for the dispatcher to select the correct suppressing control (the negative damping one or the forced oscillating one) during the oscillation period to stop the propagation of oscillations.

\section{Conflict of Interests}

The authors declare that there is no conflict of interests regarding the publication of this paper.

\section{Acknowledgment}

This work was supported by the National Natural Science Foundation of China (nos. 51177057 and 51228701).

\section{References}

[1] R. Thirumalaivasan, M. Janaki, and N. Prabhu, "Investigation of SSR characteristics of hybrid series compensated power system with SSSC," Advances in Power Electronics, vol. 2011, Article ID 621818, 8 pages, 2011.

[2] L. Chen, Y. Min, and W. Hu, "An energy-based method for location of power system oscillation source," IEEE Transactions on Power Systems, vol. 28, no. 2, pp. 828-836, 2013.

[3] A. N. Hussain, F. Malek, M. A. Rashid, L. Mohamed, and N. A. M. Affendi, "Optimal coordinated design of multiple damping controllers based on PSS and UPFC device to improve dynamic stability in the power system," Mathematical Problems in Engineering, vol. 2013, Article ID 965282, 15 pages, 2013.

[4] J. H. Chow, J. J. Sanchez-Gasca, H. Ren, and S. Wang, "Power system damping controller design," IEEE Control Systems Magazine, vol. 20, no. 4, pp. 82-90, 2000.

[5] F. P. DeMello and C. Concordia, "Concepts of synchronous machine stability as affected by excitation control," IEEE Transactions on Power Apparatus and Systems, vol. 88, no. 4, pp. 316329, 1969.

[6] A. A. Shaltout and R. T. H. Alden, "Analysis of damping and synchronizing torques-II-effect of operating conditions and machine parameters," IEEE Transactions on Power Apparatus and Systems, vol. 98, no. 5, pp. 1701-1708, 1979.
[7] A. L. S. Pereira, P. B. de Araujo, and C. T. Miasaki, "Analysis of electric torque in single machine-infinite bus system using phase shifters," IEEE Latin America Transactions, vol. 6, no. 5, pp. 389-394, 2008.

[8] Y. Zhang, D. Yue, and S. Hu, "Wide-area $H_{\infty}$ control for damping interarea oscillations with event-triggered scheme," Mathematical Problems in Engineering, vol. 2013, Article ID 517608, 11 pages, 2013.

[9] T. Hussein and A. Shamekh, "Performance assessment of fuzzy logic power system stabilizer on north benghazi power plant," Conference Papers in Engineering, vol. 2013, Article ID 635808, 6 pages, 2013.

[10] M. A. Magdy and F. Coowar, "Frequency domain analysis of power system forced oscillations," IEE Proceedings C: Generation Transmission and Distribution, vol. 137, no. 4, pp. 261-268, 1990.

[11] C. D. Vournas, N. Krassas, and B. C. Papadias, "Analysis of forced oscillations in a multimachine power system," in Proceedings of International Conference on Control, vol. 1, pp. 443-448, Edinburgh, UK, March 1991.

[12] Z.-Y. Han, R.-M. He, and Y.-H. Xu, "Power system low frequency oscillation of resonance mechanism induced by turbopressure pulsation," Proceedings of the Chinese Society of Electrical Engineering, vol. 25, no. 21, pp. 14-18, 2005 (Chinese).

[13] Y. Yang, G. Li, P. Liu, and H. Liu, "Mechanism analysis of forced power oscillation events based on WAMS measured data," in Proceedings of the 2nd Annual Conference on Electrical and Control Engineering (ICECE '11), pp. 5328-5331, September 2011.

[14] W. Zhu, Y.-Q. Zhou, X.-Y. Tan, and Y.-J. Tang, "Mechanism analysis of resonance-type low-frequency oscillation caused by networks side disturbance," Proceedings of the Chinese Society of Electrical Engineering, vol. 29, no. 25, pp. 37-42, 2009 (Chinese).

[15] W. Hu, T. Lin, Y. Gao et al., "Disturbance source location of forced power oscillation in regional power grid," in Proceedings of the IEEE Power Engineering and Automation Conference (PEAM '11), vol. 2, pp. 363-366, September 2011.

[16] X. Qiu, Z. Yu, M. Lei et al., "WAMS based statistics and assessment of low frequency oscillation in Shandong power grid," Automation of Electric Power Systems, vol. 32, no. 6, pp. 95-98, 2008 (Chinese).

[17] Y. Yu, Y. Min, L. Chen, and P. Ju, "The disturbance source identification of forced power oscillation caused by continuous cyclical load," in Proceedings of the 4th International Conference on Electric Utility Deregulation and Restructuring and Power Technologies (DRPT '11), pp. 308-313, July 2011.

[18] Y. Li, W. S. Jia, and W. F. Li, "Online identification of power oscillation properties based on the initial period of wave," Proceedings of the CSEE, vol. 33, no. 25, pp. 54-60, 2013 (Chinese).

[19] Y. Tang, "Fundamental theory of forced power oscillation in power system," Power System Technology, vol. 30, no. 10, pp. 2933, 2006.

[20] S.-F. Jiang, D.-B. Fu, and S.-Y. Wu, "Structural reliability assessment by integrating sensitivity analysis and support vector machine," Mathematical Problems in Engineering, vol. 2014, Article ID 586191, 6 pages, 2014. 
[21] B. Yan, Y. Cui, L. Zhang et al., "Beam structure damage identification based on BP neural network and support vector machine," Mathematical Problems in Engineering, vol. 2014, Article ID 850141, 8 pages, 2014.

[22] M. Ferras, C.-C. Leung, C. Barras, and J.-L. Gauvain, "Comparison of speaker adaptation methods as feature extraction for SVM-based speaker recognition," IEEE Transactions on Audio, Speech and Language Processing, vol. 18, no. 6, pp. 1366-1378, 2010.

[23] Y. J. Bao, C. J. Song, W. S. Wang, T. Yan, L. Wang, and L. Yu, "Damage detection of bridge structure based on SVM," Mathematical Problems in Engineering, vol. 2013, Article ID 490372, 7 pages, 2013.

[24] B. P. Li and M. Q. H. Meng, "Tumor recognition in wireless capsule endoscopy images using textural features and SVM-based feature selection," IEEE Transactions on Information Technology in Biomedicine, vol. 16, no. 3, pp. 323-329, 2012.

[25] H. He and J. A. Starzyk, "A self-organizing learning array system for power quality classification based on wavelet transform," IEEE Transactions on Power Delivery, vol. 21, no. 1, pp. 286-295, 2006.

[26] N. E. Huang and S. S. Shen, Hilbert-Huang Transform and Its Applications, World Scientific Press, Singapore, 1st edition, 2005.

[27] V. N. Vapnik, Statistical Learning Theory, John Wiley \& Sons, New York, NY, USA, 1998.

[28] C. Cortes and V. Vapnik, "Support-vector networks," Machine Learning, vol. 20, no. 3, pp. 273-297, 1995.

[29] D. J. Yang, J. Y. Ding, H. Q. Shao, H. P. Xu, and J. Q. Huang, "WAMS based characteristic discrimination of negative damping low frequency oscillation and Forced oscillation," Automation of Electric Power Systems, vol. 37, no. 13, pp. 57-62, 2013. 


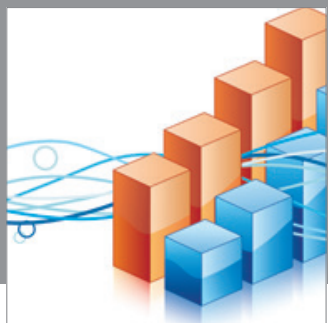

Advances in

Operations Research

mansans

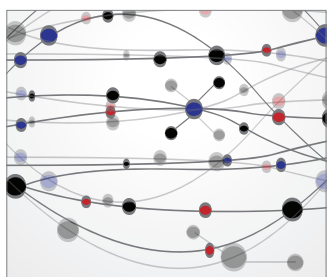

The Scientific World Journal
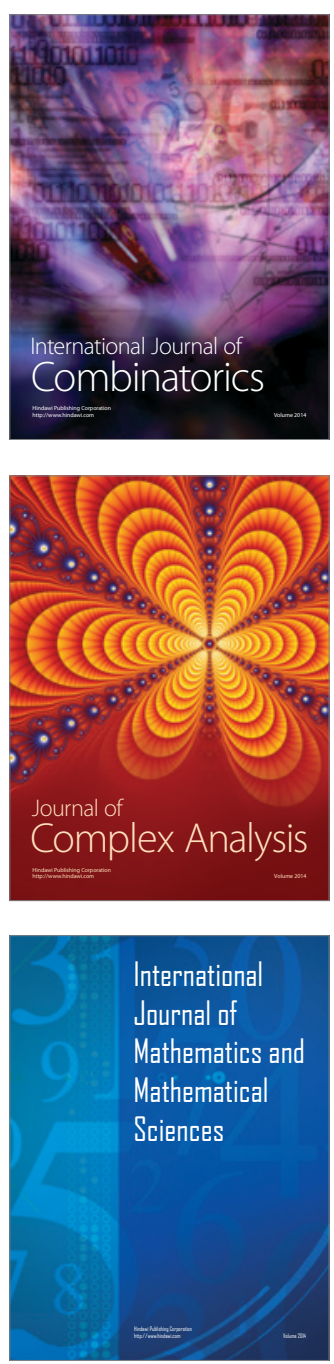
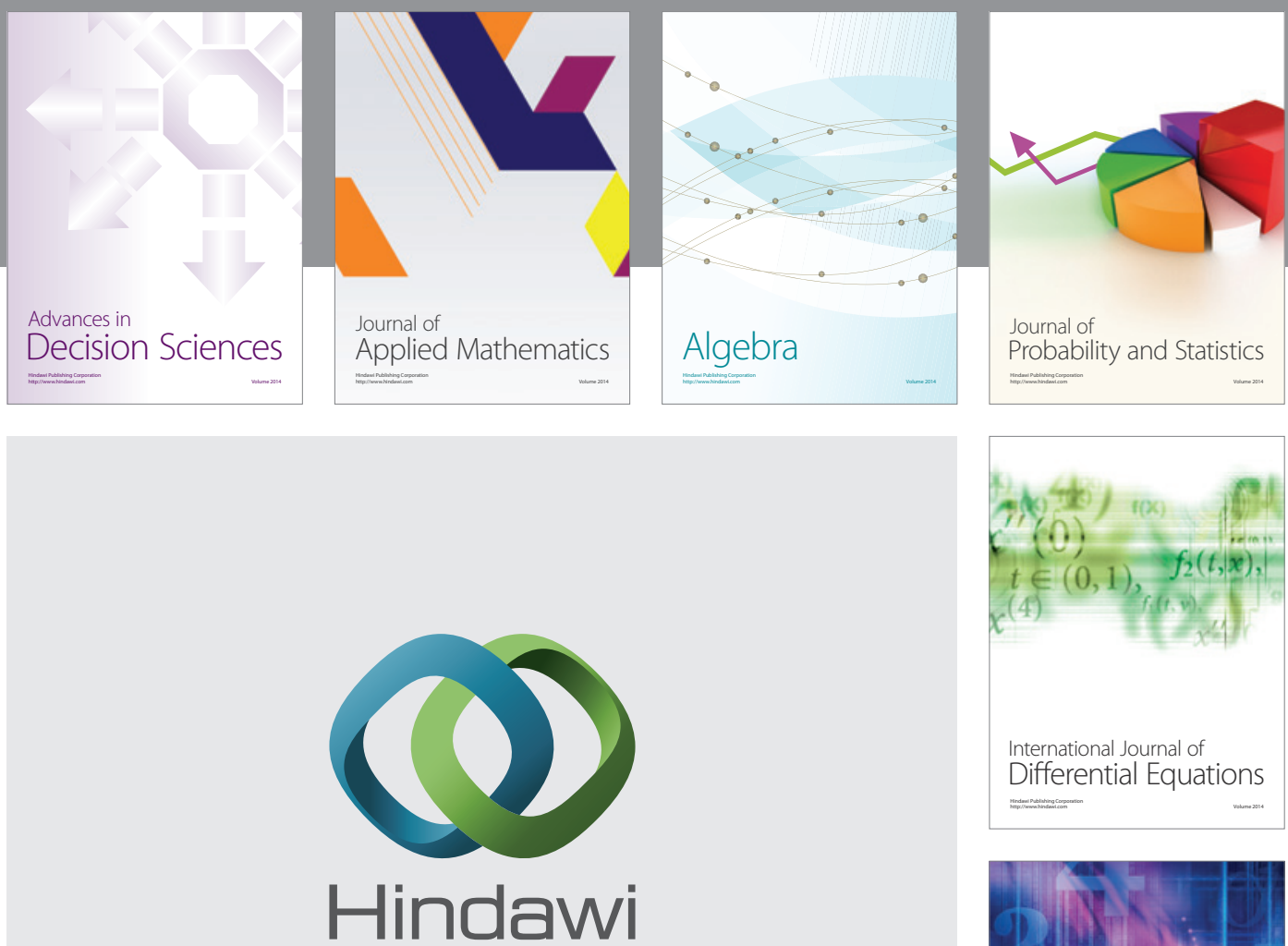

Submit your manuscripts at http://www.hindawi.com
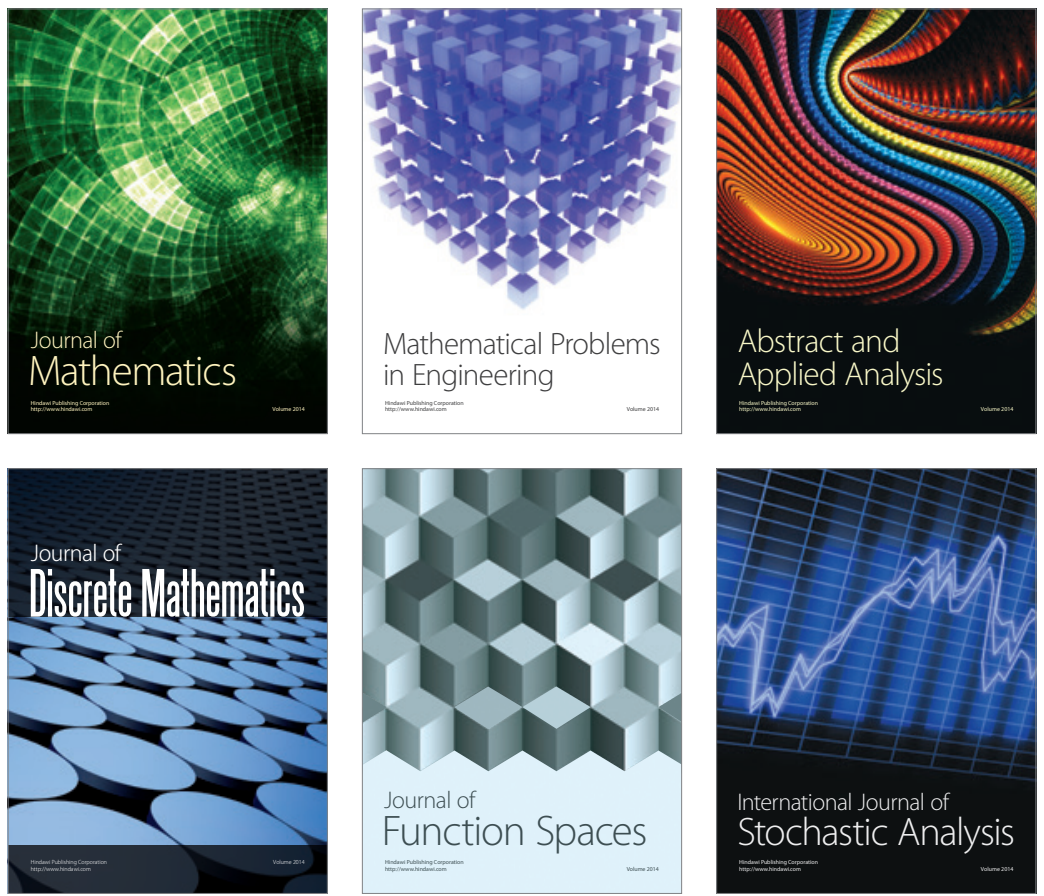

Journal of

Function Spaces

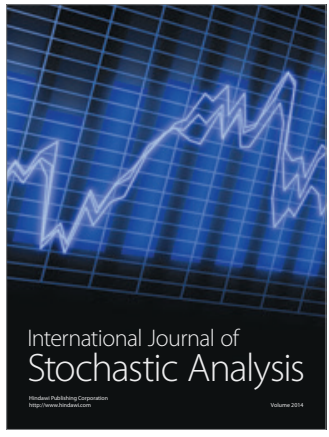

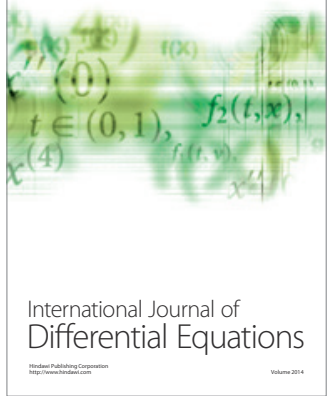
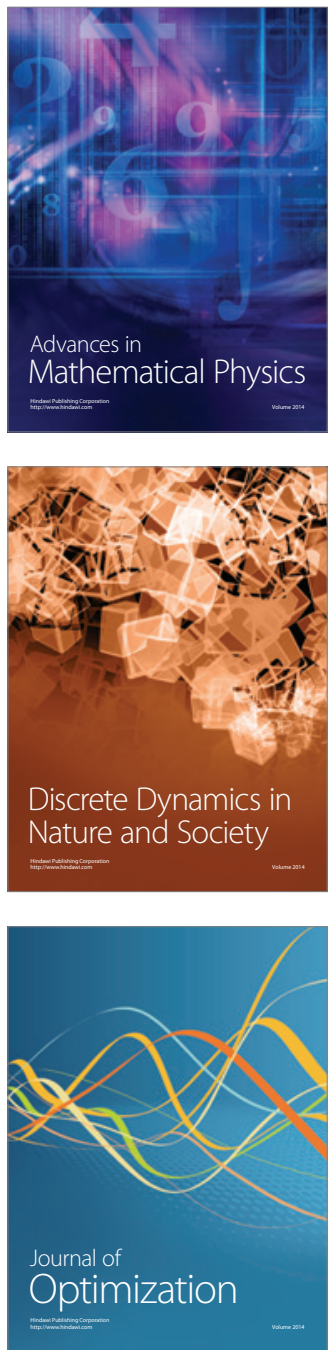